\title{
Velopharyngeal stenosis, a late complication of radiotherapy
}

- $\quad$ Siew Ching Lee

- $\quad$ Ing Ping Tang

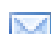

- Avatar Singh

- $\quad$ S. Selva Kumar

- $\quad$ Shashinder Singh

Received 26 April 2008; accepted 18 February 2009.

- $\quad$ Abstract

- $\quad$ Full Text

- $\underline{\text { PDF }}$

- $\quad \underline{\text { Images }}$

- References

\begin{abstract}
Choanal stenosis has recently been recognized as a late complication of radiation therapy for nasopharyngeal carcinoma. The management of velopharyngeal stenosis is challenging with high risk of restenosis. We report a case of velopharyngeal stenosis post-radiotherapy and illustrated the use of mitomycin-C to prevent restenosis. Mitomycin-C application has being shown useful adjunct to surgical technique in managing nasopharyngeal stenosis for surgeons.
\end{abstract}

Keywords: Nasopharyngeal stenosis, Velopharyngeal stenosis 